\title{
Journal of Technology and Science Education
}

\section{SELF-ASSESSMENT EXERCISES IN CONTINUUM MECHANICS WITH AUTONOMOUS LEARNING}

\author{
Jordi Marcé-Nogué, LLuís Gil, Marco A. Pérez, Montserrat Sánchez \\ Departament de Resistència de Materials i Estructures a l'Enginyeria, Universitat Politècnica de Catalunya, \\ Terrassa, Spain \\ Jordi.marce@upc.edu, Iluis.qil@upc.edu, marco.antonio.perez@upc.edu, Montserrat.sanchez@upc.edu
}

Received October 2012

Accepted November 2012

\section{Abstract}

The main objective of this work is to generate a set of exercises to improve the autonomous learning in "Continuum Mechanics" through a virtual platform. Students will have to resolve four exercises autonomously related to the subject developed in class and they will post the solutions on the virtual platform within a deadline. Students will self-correct the exercises and assign a mark according to the rubric indicated by teachers. The work presented herein is based in applying the research done in autonomous learning by the Community of Practice GRAPAU of the RIMA Project (UPC-BarcelonaTech).

Keywords - continuum mechanics, autonomous learning, virtual platform.

\section{INTRODUCTION}

University teaching has changed the curriculum and syllabus of their subjects by adjusting it to the technology evolution. Over the last decades, the increasing capabilities of computers have solved many unresolved physical problems by using a numerical and computational methodology. Up to now, many of these problems could only be solved in class by the teacher in very particular cases of simple geometries or simplifications of the formulation.

Today most university schools have implemented successful practices and modifications in the subjects to accommodate the computational resolutions. However, most of these changes have taken place at the end of the undergraduate courses when the students have gained clear vision along with practical approximation. Also it has provided the students with the necessary tools to relate their knowledge achieved previously with the usual practice of computational calculations.

The subjects included in the first cycle of undergraduate degrees, especially scientific degrees, have not experienced very much these changes since their purpose is to generate learning processes with theoretical approximation which usually are resolution procedures for problems and formula demonstrations necessary to learn scientific laws. Moreover, in higher education, university and adult teaching perspectives that have designed a learning process for subjects using skills help teachers to develop programs based on academic and professional profiles (Farrar \& Connor, 2011) and contributes to reduce the gap between the education and reality of the labour market.

One of the generic skills included in the curriculum of Escola Tècnica Superior d'Enginyeria Industrial I Aeronàutica de Terrassa (ETSEIAT) is the autonomous learning. According to the Institut de Ciències de I'Eduació (ICE) at the Universitat Politècnica de Catalunya (UPC), "Autonomous learning is the ability to detect gaps in self-knowledge and overcoming them through critical thinking and choosing the best action to extend that knowledge" (Institut de Ciències de L'Educació, 2008). However, it should be noted that people 
who learn by themselves are autodidact and, therefore, the design of autonomous learning activities must conform the guidelines provided by the teacher so that learning activities must be autonomous learning activities.

ICE recommends establishing three levels to finally reach the full competency of autonomous learning in the last period. These levels will depend on the location of the subject within the curriculum and also in the increasing difficulty (conducted, guided and autonomous) which must be taken into account when an activity is being designed (Marcé-Nogué, Salán, Aragoneses, Bernat, Escrig, Otero et al., 2012). Since in ETSEIAT the subject "Continuous Mechanics" is located just after the selective phase, we propose some activities corresponding to the level 2 (guided) of the curriculum. Although part of the activity is supervised, by the time of completion of each task and some sources of information will not be supervised any longer and the activity will be moved to level 2 .

Among all the generic skills, Autonomous Learning has an important role in the design of Course Guides using the European Credit Transfer System (ECTS) criteria because all the activities designed by the teachers must be taken into account in the schedule of the course. For this reason, UPC created the RIMA Project with the aim to become a forum to share experiences among the different communities working with innovation teaching, learning methodologies or generic research skills in engineering education (Salán, Martinez-Martinez, Portet \& Torra, 2012). The "GRup d'interés per a l'APrenentatge AUtònom" (GRAPAU) is a Community of Practice of the RIMA Project that has focused their work on collecting activities and experiences related to the Autonomous Learning. The work presented herein is based in applying the research done in autonomous learning by GRAPAU and the evaluation of the good teaching practices (Marcé-Nogué et al., 2012).

Autonomous Learning has also an important role in e-learning because either online or semi-distance courses are designed on the principles of the student autonomy. Not only face-to-face learning needs to provide students with well-designed Autonomous Learning tools research like education videos (Bravo, Amante-García, Simo, Enache \& Fernandez, 2011; Fernandez, Simo, Algaba, Albareda-Sambola, Amante-García, Enache et al., 2011; Simo, Fernandez, Algaba, Salan, Enache, Albareda-Sambola et al., 2010). Online learning will also need these tools and criteria to generate individual content (Tavangarian, Leypold, Nölting, Röser \& Voigt, 2004). Autonomous learning can also be used to complement another generic skill, for example, to create learning methodologies for team work skills (Rodríguez, Fargas, Llumà, Jorba \& Salán, 2012).

\section{METHODOLOGY}

The main purpose of this work is the generation of some exercises to improve the autonomous learning in a course of Continuum Mechanics using a virtual platform and also using a self-assessment process. It has been proved that the use of tools and specific methods as virtual platforms, self-assessment, peer-assessments, etc. optimizes the effort spent in following-up or in feedback processes which are key factors to enhance the students learning (Del Canto, Gallego, López, Medina, Monchón, Mora et al., 2011). Considering that receiving a feedback is essential to improve the performance of the learners (Garcia-Sanpedro, 2012), it will not be the only mark of the exercise. The solution of the exercise and a rubric to guide the self-assessment will be included.

To encourage the autonomous learning during the course, students will have to resolve four exercises autonomously related to the subject developed in class and they will post the solutions on the virtual platform. Each exercise has a different worksheet in which the students will have to enter the solutions by filling in the blanks provided (resolution procedure not requested). After sending their resolved exercises, the teacher will post the solutions on the virtual platform along with a rubric to facilitate the correction of the exercise. This rubric will be a document that the student must complete with the corrections. Each student must correct its own exercise, assign a mark and post it in the virtual platform before the indicated deadline (Figure 1).

At the beginning of the course the students will be given a calendar with the important dates to be considered for the completion of the exercises. This way, the students will be able to decide an appropriate schedule as well as the time to spend in each task (based on Table 1 from the spring course in 2011). Although the teacher will supervise the whole procedure, he/she will not review all the exercises submitted by each student, only some of them after being selected by an objective criteria. In case that an exercise shows something unusual during the self-assessment process, this student will be classified as a "student in audit", which means that the teacher will review all the exercises of this student.

Moodle is used as a virtual platform by the digital campus of UPC, called ATENEA (https://atenea.upc.edu/) and, consequently, is the virtual platform used in this work. Moodle (Modular Object-Oriented Dynamic Learning

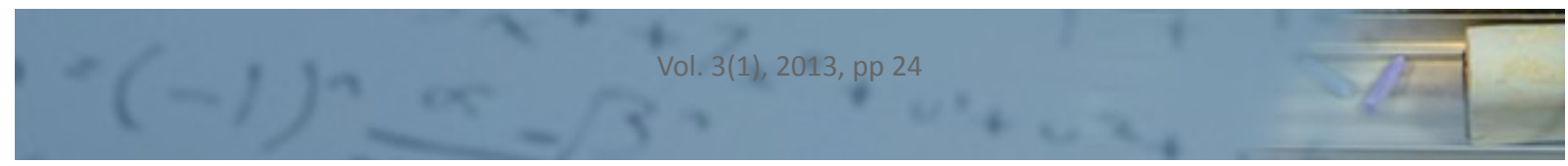


Environment) is a free source e-learning software platform, also known as a Course Management System, Learning Management System, or Virtual Learning Environment. It was developed to help educators to create online courses focused on interaction and collaborative construction of content (Al-Ajlan \& Zedan, 2008; Baskerville \& Robb, 2005).

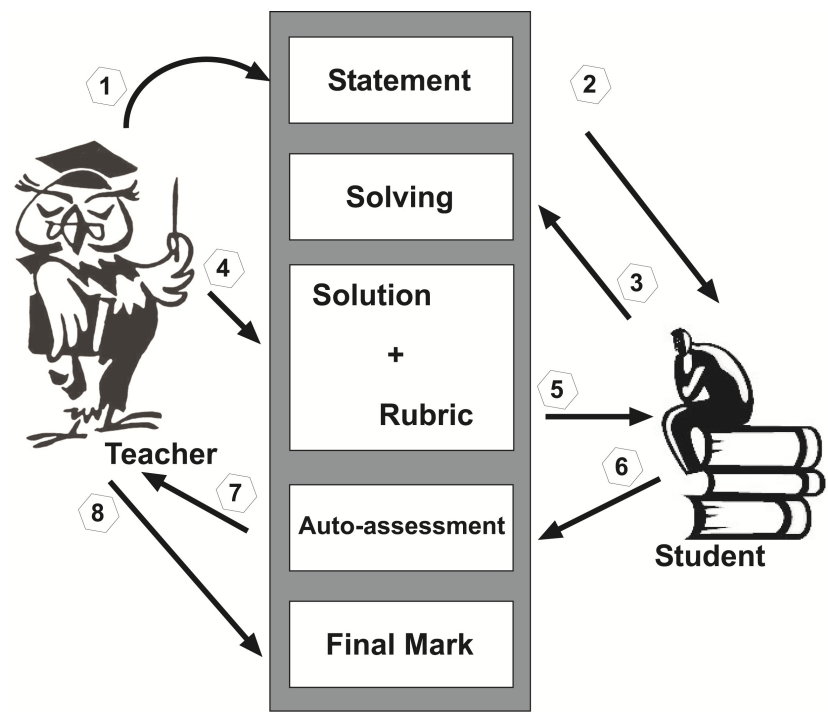

Figure 1. Flow diagram of the autonomous learning activities designed considering the interaction between the teacher and the students. The numbers indicate the order followed

\begin{tabular}{|c|c|c|}
\hline & Publication Date of the exercise & Delivery Deadline of the exercise \\
\hline Exercise 1 & March $10^{\text {th }}$ & March $18^{\text {th }}$ at $14: 00$ \\
\hline Exercise 2 & March $24^{\text {th }}$ & April $1^{\text {st }}$ at $14: 00$ \\
\hline Exercise 3 & May $5^{\text {th }}$ & May $13^{\text {th }}$ at $14: 00$ \\
\hline Exercise 4 & May 19 & May $27^{\text {th }}$ at $14: 00$ \\
\hline & Publication Date of the solution & Deliver Deadline of the rubric \\
\hline Problem 1 & March $21^{\text {st }}$ & March $25^{\text {th }}$ at $14: 00$ \\
\hline Problem 2 & April $4^{\text {th }}$ & April $8^{\text {th }}$ at $14: 00$ \\
\hline Problem 3 & May $16^{\text {th }}$ & May $20^{\text {th }}$ at $14: 00$ \\
\hline Problem 4 & May $30^{\text {th }}$ & June $3^{\text {rd }}$ at $14: 00$ \\
\hline
\end{tabular}

Table 1. Publication dates of the exercises and solutions posted on the virtual platform and delivery deadlines during the spring course in 2011

\section{SPECIFIC CONTENTS}

The proposed exercises combine several tools currently available on the internet and also start-up tools. The worksheets (Figure 2) take into account that the exercise will be corrected according to the rubric and, for this reason, the results will be written in the gap allocated to each question. Students will indicate the solution without the resolution procedure. 


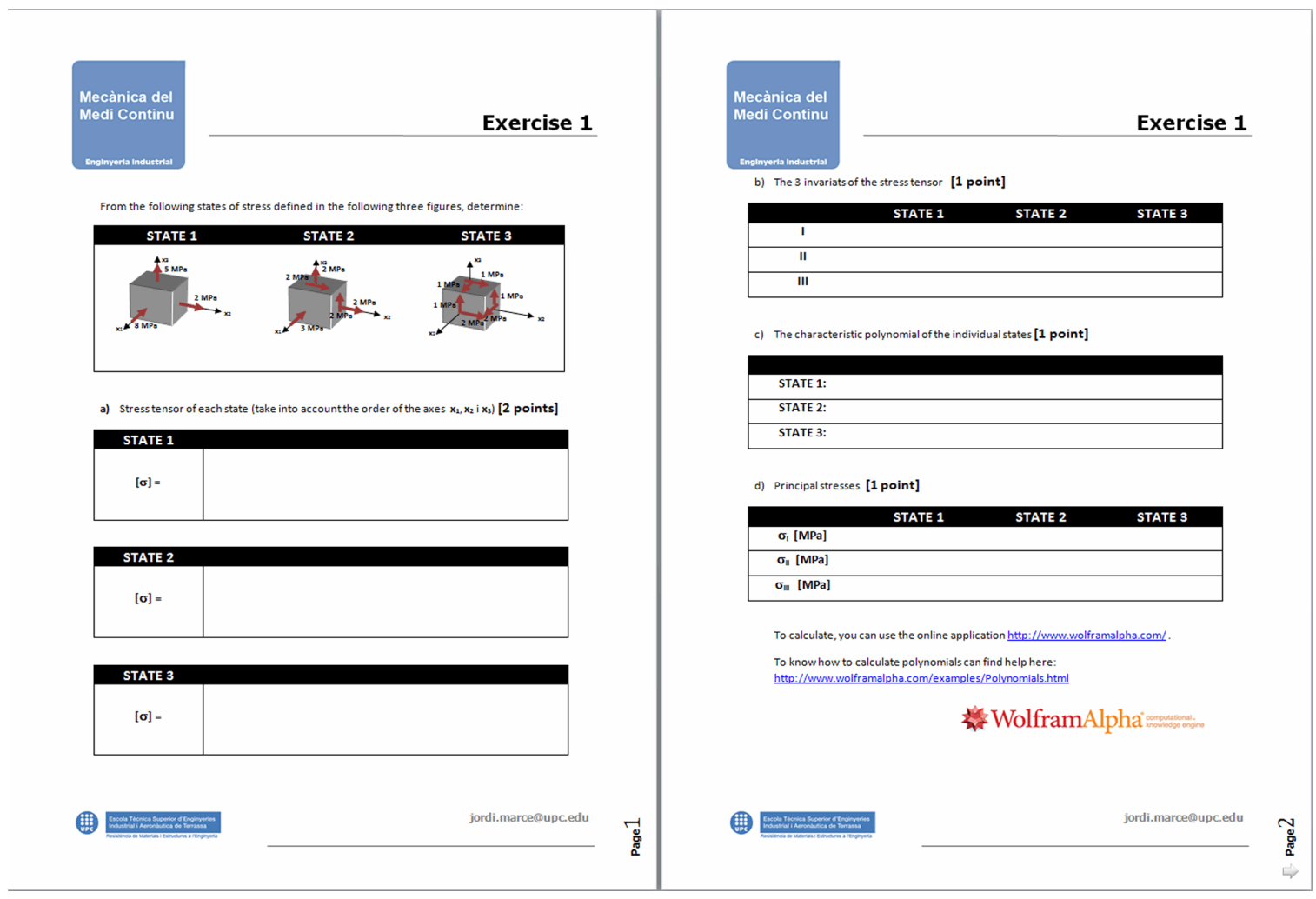

Figure 2. Worksheet of Exercise 1 with the blanks to be filled in with the results

\subsection{Exercise 1}

It is an exercise with a defined stress in which states should determine the tensor of stress (analytically) and the Mohr circles (graphically) in order to compare the coincidence of the analytical and the graphical results. Similar problems have been solved previously in class when the students were given the necessary mathematical tools and had the help of a scientific calculator. In this exercise two different on-line tools have been offered: An application of advanced mathematical tools and another application where the Mohr circles can be solved graphically.

The application http://www.wolframalpha.com/ is suggested to resolve the analytical part. Wolfram Alpha is an online engine of responses that answers factual queries directly by computing the answer from structured data, rather than providing a list of documents or web pages with possible answers. Wolfram Alpha is based on the computational platform Mathematica. In this case, the student needs to solve a third-order polynomial which can be done easily by following the instructions of the help book also available online.

The JavaScript web application http://www.engapplets.vt.edu/Mohr/iava/nsfapplets/MohrCircles23D/Applets/applet.htm is proposed for the resolution of the graphical part which can easily solve the Mohr circles interactively "Mohr's Circles for 3-D Stress Analysis". Finally, students must comment the results obtained by both methods of resolution, which a priori need to be matched.

\subsection{Exercise 2}

Computational mechanics is the discipline that uses computational methods to study phenomena governed by the principles of mechanics (Buchanan, 1990). Rather than a sub-discipline of applied mechanics, nowadays it is considered as the "third way" besides theoretical and experimental sciences and it is important that the student become familiar with this discipline.

In this case, it is an exercise where the computational methods, that can solve the stress state, are introduced to the students. The student don't know the computational methods, since they are not part of the agenda, but they must know how to interpret the results and must reach the conclusion that computational methods are an alternative to the complex geometries of the problems being solved in class. The students will work previously

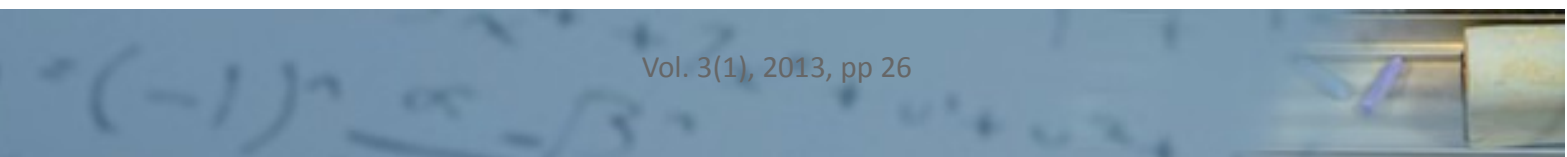


in class a similar exercise to learn the keys to interpret the results of a stress state obtained by computational methods.

\subsection{Exercise 3}

In this exercise there is a simple geometry submitted to a certain stress state that the students must solve in a parallel way using analytical methods, as the Airy function, and also computational methods. Since the Finite Element Method (FEM) is not included in the contents of the course and the use of a package of Finite Element Analysis (FEA) would require a considerable number of training hours (Zienkiewicz, 1971), the procedure of the exercise will be as follows:

1. The student starts the exercises by doing a search in www.wikipedia.com about computational mechanics, the MEF and FEA in order to synthesize content of the theoretical topics.

2. Provide the computational resolution solved by using a FEA video. The student only has to view it to understand how a FEA procedure works in a commercial package. www.youtube.com/watch? v=NDRBvgVab9o

3. Provide the students with a video review of the methodology used to solve problems using Airy functions in a simple geometry in order to review the topics covered in class: www.youtube.com/watch?v=kdQJ|Bo6m50. Finally, to solve a problem of the same type to check the methodology.

The educational videos created here are called "low-cost video" because, as (Fernandez et al., 2011) define, they are a short demonstration stream videos with a very specific goal created in a very short period of time with few resources and they can be combined or embedded within other course materials. The work (Simo et al., 2010) reveals that when the students use low-cost educational videos the student motivation improves and increases the perceived efficiency upon the learning and teaching process. The video-sharing website Youtube is used as a video repository.

\subsection{Exercise 4}

It is an exercise to review different topics of continuum mechanics related to stress, strain and constitutive laws in large deformations. This exercise does not include on-line tools or teaching videos.

\section{RESULTS}

A survey among the students was carried out to find out the satisfaction level of the exercises and identify potential gaps in them. The survey was created online using questionnaires offered by Google (https://docs.google.com/). Google Docs is a free, web-based office suite and data storage service offered by Google. It allows users to create and edit documents online combining the features of write a spreadsheet. The results of the surveys can be managed in a spreadsheet and allows embed code "html" in the virtual platform, facilitating an asynchronous participation of the students. The questions included in the survey of the students were the following:

1. The exercises correspond to the course syllabus

2. The level of difficulty of the exercises

3. The time spent in each exercise

4. The self-assessment system helps you to be more aware of errors and therefore improves your learning with no need of teacher corrections

5. The content of the rubric is understandable and facilitates the correction of the exercises

6. The evaluation system of the exercises is fair and appropriate

7. The virtual platform used as a system to deliver the exercises is easy to use

8. The content of the subject is consolidated after doing the exercises and their corrections

The questions are evaluated within a scale from 1 to 5 (from totally disagree to totally agree) except for questions 2 and 3 where the scale from 1 to 5 goes from very low to very high. In the spring semester of the academic year 2010/2011, 65 students out of 95 answered the survey (68\%). The results are shown in Figure 3.

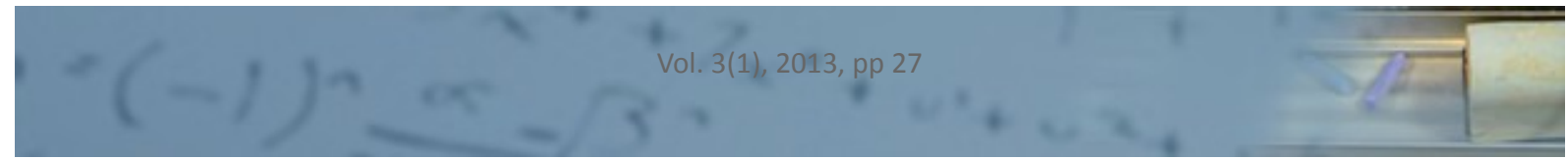




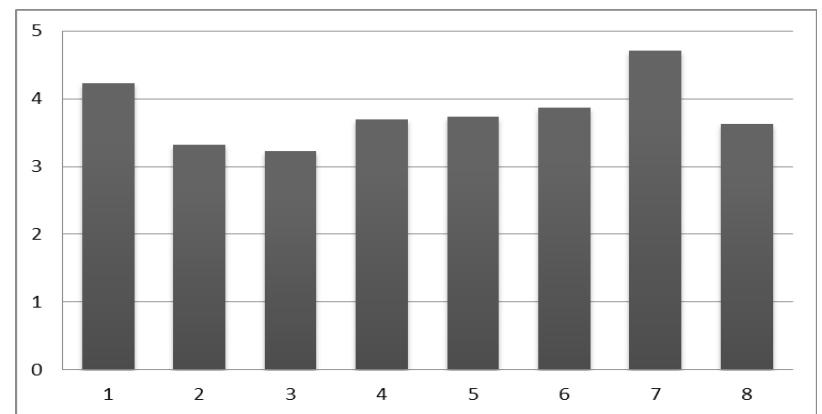

Figure 3. Results of the survey during the spring semester of 2011/12 academic year

Students evaluated positively the following issues: The exercises correspond to the course syllabus, the selfassessment system helps you to be more aware of errors and therefore improves your learning with no need of teacher corrections, the content of the rubric is understandable and facilitates the correction of the exercises, the evaluation system of the exercises is fair and appropriate, the virtual platform used as a system to deliver the exercises is easy to use and the content of the subject is consolidated after doing the exercises and their corrections. As per the time and difficulty, students consider that the level of difficulty of the exercises and the time spent to each one is the appropriated.

The fundamental aspects (Marcé-Nogué et al., 2012) that an activity must consider or contain for an adequate autonomous learning are classified in the following four groups: LOAD: The load of work to be performed by the students and the teacher in the activity must be balanced. MATURITY: It is necessary to place the activity in the proper context of the subject and the degree. EFFECTIVENESS: It must be an indicator for teachers and students to measure the student learning progress. PROTOCOL: The activity must be a guided and structured task.

To evaluate the exercises as a good-practice in autonomous learning, the Load, Maturity, Effectiveness and Protocol of the activities are evaluated by the teacher assistant (Table 2) considering that the ideal case would be scored with 5 in each aspect. According to the teacher assistant, the Load is not considered balanced enough, but instead, the Maturity, Effectiveness and Protocol achieve the desired results.

\begin{tabular}{|l|r|r|r|r|}
\hline & Teacher 1 & \multicolumn{1}{|c|}{ Teacher 2 } & \multicolumn{1}{c|}{ Teacher 3 } & \multicolumn{1}{c|}{$\begin{array}{c}\text { Truncated } \\
\text { Average } \\
\text { Qualification }\end{array}$} \\
\hline Load & 2 & 2 & 4 & 2 \\
\hline Maturity & 5 & 5 & 5 & 5 \\
\hline Effectiveness & 4 & 5 & 4 & 4 \\
\hline Protocol & 4 & 5 & 5 & 4 \\
\hline Total Points & 15 & 17 & 18 & 15 \\
\hline
\end{tabular}

Table 2. Evaluation of the fundamental aspects (Load, Maturity, Effectiveness and Protocol)

of the autonomous learning activities

\section{CONCLUSIONS}

According to the evidences of the student's survey and the evaluation of the fundamental aspects carried out by the teachers, the self-assessment exercises presented herein are positively assessed. Students were asked in the online survey about the content of the exercises, the methodology and the process of self-assessment and they considered that the activities are suitable for the autonomous learning of continuum mechanics.

It is important to remark that the self-assessment methodology described is a good procedure to improve autonomous learning. As described above, students will indicate the solution without posting the resolution procedure of the exercises and they will be corrected according to the given rubric. According to the survey, students consider that the content of the subject is consolidated after completion of the exercises and their corrections.

In spite of this general conclusion, the evaluation of the fundamental aspects performed by the teachers in table 2 reflects that some effort has to be made to improve the balance between the workload performed by the students and the teacher. An additional benefit for teachers is the reduction of time spent in routine

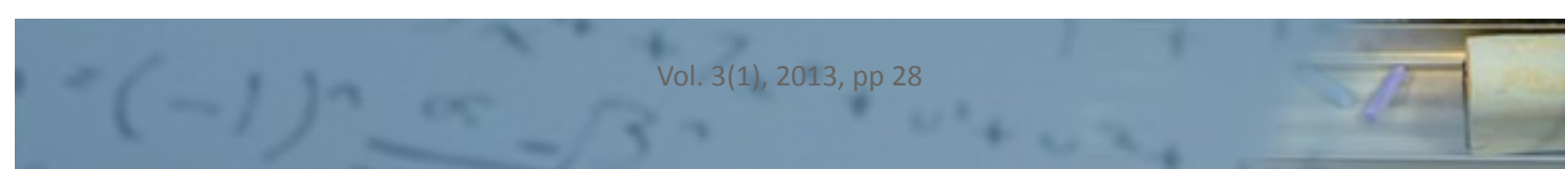


corrections which now can be used for more profitable teaching activities. Although the activity has not achieved the optimal autonomous learning level, the evaluation of Maturity, Effectiveness and Protocol reached the consideration of a good-practice in autonomous learning.

\section{ACKNOWLEDGEMENTS}

We especially thank Sonia Segura (UPC, Terrassa) for her assistance in designing the figures and Marilà Lucas (UPC, Terrassa) for the English language supervision. This study has been supported by mean of projects "Millora de la Qualitat docent" (MQD 2009) of Agència de Gestió d'Ajuts Universitaris i de Recerca (AGAUR). The work is also included in the research in autonomous learning of "GRup d'interés per a l'APrenentatge AUtònom" (GRAPAU) from the RIMA Project.

\section{REFERENCES}

Al-Ajlan, A. \& Zedan, H. (2008). Why Moodle. 12th IEEE International Workshop on Future Trends of Distributed Computing Systems, 58-64. Inst. of Elec. and Elec. Eng. Computer Society. http://dx.doi.org/10.1109/FTDCS.2008.22

Baskerville, B. \& Robb, T. (2005). Using Moodle for teaching. Review Literature And Arts Of The Americas, 1(1), 138-151.

Bravo, E.R., Amante-García, B., Simo, P., Enache, M. \& Fernandez, V. (2011). Video as a new teaching tool to increase student motivation. 2011 IEEE Global Engineering Education Conference (EDUCON), 638-642. http://dx.doi.org/10.1109/EDUCON.2011.5773205

Buchanan, G.R. (1990). Computational Mechanics. Computational Mechanics, 32(1), $167-172$. http://dx.doi.org/10.1146/annurev.matsci.32.103101.153157

Del Canto, P., Gallego, I., López, J.M., Medina, E., Monchón, F., Mora, J. et al. (2011). Follow-Up and Feedback Processes in the Ehea. Journal of Technology and Science Education, 1(1), 12-22. http://dx.doi.org/10.3926/jotse.2011.14

Farrar, R., \& Connor, P. O. (2011). What is Autonomous Learning ? The Role of Autonomous Learning in Higher $\begin{array}{lllll}\text { Education. } \quad \text { Discourse, } & \text { (April), } & \text { Retrieved } & \text { from }\end{array}$ http://www.prs.heacademy.ac.uk/pdf.html/PrsDiscourseArticles/113

Fernandez, V., Simo, P.E.P., Algaba, I., Albareda-Sambola, M., Amante-García, B., Enache, M. et al. (2011). LowCost Educational Videos for Engineering Students: a new Concept based on Video Streaming and YouTube Channels. International Journal of Engineering Education, 27(3), 518-527.

Garcia-Sanpedro, M. (2012). Feedback and Feedforward: Focal points for improving academic performance. Journal of Technology and Science Education, 2(2), 77-85.

Institut de Ciències de L'Educació. (2008). Quadern per treballar les competències genèriques a les assignatures: Aprenentatge autònom. Barcelona. Retrieved from http://ben.upc.es/documents/eees/196/frameset.html

Marcé-Nogué, J., Salán, N., Aragoneses, A., Bernat, E., Escrig, C., Otero, B. et al. (2012). Teaching Engineering with Autonomous Learning Tools: Good Practices in GRAPAU-RIMA. Procedia - Social and Behavioral Sciences, 46, 629-634. http://dx.doi.org/10.1016/j.sbspro.2012.05.175

Rodríguez, D., Fargas, G., Llumà, J., Jorba, J. \& Salán, M.N. (2012). Learning Experiences of the GidMAT-RIMA Group with Materials Engineering Students in Autonomous Learning and Working in Teams Generic Skills. Procedia - Social and Behavioral Sciences, 46, 4369-4373. http://dx.doi.org/10.1016/j.sbspro.2012.06.256

Salán, M.N., Martinez-Martinez, M., Portet, E. \& Torra, I. (2012). RIMA Project: Activities and Initiatives Communion and Sharing in Educational Innovation at UPC-BARCELONATECH. Procedia - Social and Behavioral Sciences, 46, 2284-2288. http://dx.doi.org/10.1016/j.sbspro.2012.05.471

Simo, P., Fernandez, V., Algaba, I., Salan, N., Enache, M., Albareda-Sambola, M. et al. (2010). Video stream and teaching channels: quantitative analysis of the use of low-cost educational videos on the web. Procedia-Social and Behavioral Sciences, 2(2), 2937-2941. http://dx.doi.org/10.1016/j.sbspro.2010.03.444 
Citation: Marcé-Nogué, J., Gil, LL., Pérez, M.A. \& Sánchez, M. (2013). Self-assessment exercises in continuum mechanics with autonomous learning. Journal of Technology and Science Education (JOTSE), 3(1), 23-30. http://dx.doi.org/10.3926/jotse.57

On-line ISSN: 2013-6374 - Print ISSN: 2014-5349 - DL: B-2000-2012

\section{AUTHORS BIOGRAPHY}

\section{Jordi Marcé-Nogué}

Departament de Resistència de Materials i Estructures a l'Enginyeria, Universitat Politècnica de Catalunya, Terrassa, Spain

PhD degree in Structural Analysis from Universitat Politècnica de Catalunya in 2009. Since 2006 he has being an assistant teacher and researcher at the Universitat Politècnica de Catalunya. He has written 10 international publications, 23 conference contributions and leaded 2 competitive research projects in educational innovation.

\section{LLuís Gil}

Departament de Resistència de Materials i Estructures a l'Enginyeria, Universitat Politècnica de Catalunya, Terrassa, Spain

Dr. Civil Engineer (1997). Assistant professor of Continuum Mechanics, Engineering Structures and Composite Materials. Principal investigator of 13 competitive research projects, 26 int. publications, 73 conference contributions, 1 patent co-authoring. Research interests: testing structures, masonry, concrete and composites.

\section{Marco A. Pérez}

Assistant Professor at the UPC where he obtained a PhD in Structural Analysis in 2012. He has a MSc degree in Mechanical Engineering and a degree in Music at the College of Music of Catalunya. During 2010 he spent a research period at the University of Colorado in Boulder (USA). His research activity is focused on composite materials, plate and shells finite elements, dynamic analysis, musical acoustics and nondestructive testing.

Departament de Resistència de Materials i Estructures a l'Enginyeria, Universitat Politècnica de Catalunya, Terrassa, Spain

\section{Montserrat Sánchez}

Departament de Resistència de Materials i Estructures a l'Enginyeria, Universitat Politècnica de Catalunya, Terrassa, Spain

Dr Mechanical Engineer and professor at the Universitat Politècnica de Catalunya. She has participated in several national projects and agreements with companies. She is currently directing two doctoral theses, has participated in 12 competitive research projects ( 2 of them as principal investigator), has conducted 14 international publications and 10 conference contributions.

Published by OmniaScience (www.omniascience.com)

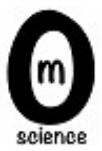

Journal of Technology and Science Education, 2013 (www.jotse.org)

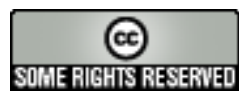

Article's contents are provided on a Attribution-Non Commercial 3.0 Creative commons license. Readers are allowed to copy, distribute and communicate article's contents, provided the author's and Intangible Capital journal's names are included. It must not be used for commercial purposes. To see the complete licence contents, please visit http://creativecommons.org/licenses/by-nc/3.0/es/ 